\title{
需求可拆分下城轨关联的公交接驳线网优化
}

\author{
邓连波，何 渊，曾俊豪，周文梁
}

中南大学交通运输工程学院, 湖南长沙 410075

摘 要：基于典型的公交接驳线网优化问题, 将每一公交站点具有唯一线路服务这一假设扩充到每一 个公交站点可由多条公交线路服务，使公交站点与公交接驳线路间具有多对多的关系，即拓展到需求在多 条线路间具有可拆分性的情形，形成需求可拆分的公交接驳线网优化问题. 依据该情形下的公交接驳线网 特性构建约束条件，分析换乘网络上乘客出行费用，并构造客流选择函数，建立需求可拆分条件下的接驱 线网优化模型。通过在遗传算法中引入公交重复站点生成和插入策略，求解该模型，算例结果表明，需求 可拆分情形下，公交接驳线网优化问题的求解时间显著增长，允许公交线路间具有重复站点可改善公交线 网, 随着网络客流量的上升, 重复站点数量增加并集中于客流较多的站点.

关键词：公共交通；公交接驳线网；需求可拆分；换乘网络；线路重复站点；遗传算法

中图分类号：U293 文献标志码：A doi：10.3724/SP. J. 1249.2020.02121

\section{Optimal design of feeder-bus network with split delivery}

\section{DENG Lianbo, HE Yuan, ZENG Junhao, and ZHOU Wenliang}

School of Traffic and Transportation Engineering, Central South University, Changsha 410075, Hunan Province, P. R. China

\begin{abstract}
Based on the typical optimization problem of the feeder-bus network design problem (FBNDP), we discuss the feeder-bus network design problem with split delivery (FBNDP-SD). The passenger demand of each bus stop is allowed to be served by different feeder-bus routes, which means that the passenger demand of each bus stop can be distributed into more than one route. Thus the relationship between bus stops and feeder-bus routes changes into many-to-many pattern. According to the characteristic of the FBNDP-SD, this paper introduces new constraints, analyzes travel cost of passengers, constructs the passenger flow selection function and establishes optimal feeder-bus network under the condition of demand splitting. We design the modified genetic algorithm and introduce repeated bus stop generating and inserting strategies. The results show that the computation time increases significantly under FBNDP-SD, and permission of repeated bus stop in feeder-bus routes has influence on feeder-bus network improvement. The amount of repeated bus stop increases as the network traffic increases, and most of these bus stops have great passenger demand.
\end{abstract}

Key words: public transport; feeder-bus network; split delivery; transfer network; repeat bus stop; genetic algorithm

Received: 2018-12-04; Accepted: 2019-03-28

Foundation: National Natural Science Foundation of China (71471179, U1834209)

Corresponding author: Professor DENG Lianbo. E-mail: lbdeng@ csu.edu.cn

Citation: DENG Lianbo, HE Yuan, ZENG Junhao, et al. Optimal design of feeder-bus network with split delivery [J]. Journal of Shenzhen University Science and Engineering, 2020, 37(2): 121-129. (in Chinese) 
在城市公共交通系统中，城市轨道交通与常规 公交之间的有效衔接，对于充分发挥公共交通的网 络化优势具有重要意义. 为此, 在进行公交线网设 计时, 需要充分考虑轨道交通线路的走向和站点分 布，由此形成公交接驳线网问题 (feeder-bus network-design problem, FBNDP), 即针对存在的一个 轨道交通系统，设计对应的公交接驳线路网络，使 之形成一个整体的接驳换乘系统. FBNDP 通过接驳 车站、线路经由及开行频率 ${ }^{[1]}$ 确定城市公交接驳线 路方案.

$\mathrm{KUAH}$ 等 $^{[1]}$ 提出，由于 FBNDP 包含旅行商问题 且目标函数具有非线性，是一个典型的多项式复杂 程度的非确定性问题，即 NP 难 (non-deterministic polynomial hard, NP-hard) 问题，适于采用启发式算 法求解. BYRNE 等 ${ }^{[2]}$ 对 $\mathrm{KUAH}$ 等 ${ }^{[1]}$ 建立的公交站 点和轨道车站间 “多对一” 的规划模型进行求解, 并提出 FBNDP. LENSTRA 等 ${ }^{[3]}$ 验证了遗传算法对 于求解类似于 FBNDP 这种包含多个变量和多个约 束的 NP 难问题，具有较高的适应度和匹配性. KUAN 等 ${ }^{[4]}$ 也通过遗传算法得到 FBNDP 较好的解 形式. 邓连波等 ${ }^{[5]}$ 考虑公交站点与轨道车站 “多对 多” 情形，建立基于换乘网络的接驳线网优化模 型. 近年来, 针对公交接驳线网优化问题, 许多学 者采用遗传算法进行求解. $\mathrm{LI}$ 等 ${ }^{[6]}$ 提供可以降低乘 客到达接驳公交成本的接驳公交调度模型，并利用 遗传算法求解大型线网. SUN 等 ${ }^{[7]}$ 以搜寻基于总出 行时间最小化的接驳公交路径和时刻表为目标，构 建针对个人需求的定制公交优化模型. ANASTASIOS 等 ${ }^{[8]}$ 采用遗传算法求解需求响应的接驳公交 最后一公里问题. TAPLIN 等 ${ }^{[9]}$ 构建基于最短步行 距离到达接驳公交车站的模型，并通过遗传算法搜 寻最大需求公交站点. 针对 FBNDP, 研究多将公交 站点与轨道车站之间的关系模式分为 “多对一” 和 “多对多” 的情形讨论，但公交站点仍限制在仅允 许被单一线路服务.

对于与 FBNDP 问题类似的开放式车辆路径问 题，如何构造满足需求可拆分的解是解决此类问题 的关键. 研究已提出一些求解方法 ${ }^{[10]}$, 如列生成 算法 ${ }^{[11]}$ 、禁忌搜索算法 ${ }^{[12-14]}$ 等.

本研究受需求可拆分条件下车辆路径问题的启 发，针对经典 FBNDP 中每一个公交站点只被单一 接驳线路服务的限制问题，将其扩充至每个公交站 点可以被多条公交接驳线路服务，即公交站点和公 交接驳线路间具有 “多对多” 的关系，使之更符合
实际客流分布规律. 由此形成需求可拆分的公交接 驳线网优化问题（feeder-bus network-design problem with split delivery, FBNDP-SD). 通过分析换乘网络 总费用，建立需求可拆分情形下的公交接驳线网优 化模型，针对此问题设计改进遗传算法，并分析讨 论不同公交站点客流需求强度下的优化结果.

\section{1 公交接驳线网描述}

经典 FBNDP 满足以下假设：(1) 每一个公交站 点只由一条公交接驳线路服务; (2) 每一条公交接 驳线路只连接到一个轨道接驳车站; (3) 所有公交 接驳线路的速度和能力统一; (4) 每一公交接驳线 路在其上的每一公交站点停靠.

满足假设(1)和(2)时，公交站点和公交线路间是 多对一情形, 与实际接驳线网存在较大差别. 为 此，取消假设(1)，即允许每一个公交站点被多条公 交接驳线路服务，将该问题拓展至需求在多条线路 间具有可拆分性的情形，形成 FBNDP-SD.

FBNDP-SD 接驳线网相关的基础网络包括 $I$ 个 常规公交站点和 $J$ 个轨道交通车站，记公交站点集 合为 $B=\{1,2, \cdots, I\}$, 轨道车站集合为 $T=\{I+1$, $I+2, \cdots, I+J\}$, 网络节点集合 $N=B \cup T$. 记任意 节点 $i 、 j$ 间的距离为 $L_{i j}, i, j \in N$.

接驳线网 $\Omega$ 由 $K$ 条公交接驳线路构成，第 $k(k=1,2, \cdots, K)$ 条公交接驳线路表示为 $\omega_{k}=\left\{n_{1}^{k}\right.$, $\left.n_{2}^{k}, \cdots, n_{P_{k}-1}^{k}, n_{P_{k}}\right\}$, 其中, 接驳车站 $n_{P_{k}} \in T$, 线路经 由公交站点 $n_{1}^{k}, n_{2}^{k}, \cdots, n_{P_{k-1}}^{k} \in B$. 公交车的平均旅行 速度为 $v_{B}$, 轨道列车的旅行速度为 $v_{T}$. 接驳线路 $k$ 的 开行频率为 $f_{k}$, 即所研究时段内接驳公交线路的开 行趟次数. 公交站点与轨道车站间的客流需求量表 示为 $P_{i d}$, 其中, $i \in B, d \in T$.

\section{FBNDP-SD 的优化建模}

\subsection{FBNDP-SD 的构成约束}

定义 $X_{i h k}$ 及 $Y_{i j k}$ 表示站点间、站点和线路间关系： $X_{i h k}=\left\{\begin{array}{cc}1, & i \text { 和 } h \text { 在第 } k \text { 条接驳线路上相邻时 } \\ 0, & \text { 其他 }\end{array}\right.$ $i=1,2, \cdots, I+J ; h=1,2, \cdots, I+J ; k=1,2, \cdots, K$. $Y_{i j k}=\left\{\begin{array}{cc}1, & i \text { 和 } j \text { 在同一条接驳线路 } k \text { 上时 } \\ 0, & \text { 其他 }\end{array}\right.$ $i=1,2, \cdots, I ; j=I+1, \cdots, I+J ; k=1,2, \cdots, K$. 


$$
\text { 当 }\left(\sum_{h=1}^{I+J} X_{i h k}+\sum_{h=1}^{I} X_{h i k}\right)\left(\sum_{h=1}^{I+J} X_{j h k}+\sum_{h=1}^{I} X_{h j k}\right)>0
$$

时, $Y_{i j k}=1$.

FBNDP-SD 线网需要满足以下网络结构约束:

$$
\begin{aligned}
& \sum_{i \notin H} \sum_{h \in H} \sum_{k=1}^{K} X_{i h k} \geqslant 1 \\
& \sum_{i=1}^{I} \sum_{j=I+1}^{I+J} X_{i j k}=1, k=1,2, \cdots, K \\
& \sum_{i=I+1}^{I+J} \sum_{h=1}^{I+J} X_{i h k}=0, k=1,2, \cdots, K \\
& \sum_{i=1}^{I} \sum_{j=1}^{I+J} X_{i j k} \geqslant 1, k=1,2, \cdots, K \\
& \sum_{k=1}^{K} \sum_{h=1}^{I+J} X_{i h k} \geqslant 1, i=1,2, \cdots, I
\end{aligned}
$$

$\sum_{p=1}^{I} X_{p i k} \leqslant 1, i=1,2, \cdots, I ; k=1,2, \cdots, K$

$\sum_{h=1}^{I+J} X_{i h k}-\sum_{p=1}^{I} X_{p i k} \geqslant 0$,

$$
i=1,2, \cdots, I ; k=1,2, \cdots, K
$$

$\sum_{h=1}^{I+J} X_{i h k}+\sum_{p=1}^{I} X_{p j k}-Y_{i j k} \leqslant 1, i=1,2, \cdots, I ;$

$j=I+1, I+2, \cdots, I+J ; k=1,2, \cdots, K$

$$
\begin{aligned}
\sum_{k=1}^{K} P_{i d}^{k}=P_{i d}, i=1,2, \cdots, I ; \\
d=I+1, I+2, \cdots, I+J
\end{aligned}
$$

$\sum_{i=1}^{I} \sum_{d=I+1}^{I+J} P_{i d}^{k} \sum_{h=1}^{I+J} X_{i h k} \leqslant f_{k} C \rho, k=1,2, \cdots, K$

$$
\sum_{k=1}^{K} f_{k} \sum_{i=1}^{I} \sum_{h=1}^{I+J} L_{i h} X_{i h k} \leqslant \frac{1}{2} N v_{B} t
$$

上述网络结构约束中, 式 (1) 保证 FBNDP-SD 线网的连通性, 即公交站点集合须通过接驳线路, 或通过其他公交站点接续至轨道交通车站. 其中, $H$ 为所有接驳轨道交通车站和部分公交站点的集 合, $H$ 为 $N$ 的子集.

式(2) 至式 (4) 保证每条接驳线路的完整性, 式(2)表示每条公交接驳线路仅连接 1 个轨道交通 车站; 式(3)表明接驳线路均终止于轨道交通车站; 式(4)限定接驳线路至少应包含 1 个公交站点和 1 个轨道交通车站.

式 (5) 至式 (8) 为接驳线网中线路与车站的关 系约束, 式(5) 表示需求可拆分情形下, 每一公交
站点至少有 1 条接驳线路经由; 式(6)至式(7)表 示同一线路上单个站点仅停靠 1 次, 且该线路必须 是一个无环的简单路; 式 (8) 是接驳线路 $k$ 与站点 $i 、 j$ 间的关系变量 $Y_{i j k}$ 约束.

式(9)表示站点需求在线路间的流量守恒约束， 其中, 客流需求 $P_{i d}$ 在接驳线路 $k$ 上的客流量表示为 $P_{i d}^{k}$. 式 (10) 表示每条接驳线路 $k$ 的开行频率 $f_{k}$ 应满 足该线路上客流的输送能力要求, 其中, $C$ 为单位 公交车的载客量; $\rho$ 为席位利用率. 式 (11) 限制线 网内公交车辆运营的总里程, 其中, $t$ 为公交车全日 可运营时间; $N$ 为该时段可投人运营的公交车辆数.

与 FBNDP 线网相比, FBNDP-SD 线网将式 (5) 中每一公交站点仅有 1 条线路经由变更为允许多于 1 条线路经由; 引人接驳线路客流量 $P_{i d}^{k}$, 增加如式 (9) 的站点需求在线路间的流量守恒约束, 并对式 (10) 的开行频率约束进行相应调整. 为与 FBNDP 线网相区别, 对式(5)中, 满足 $\sum_{k=1}^{K} \sum_{h=1}^{I+J} X_{i h k}>1$ 的站 点, 称之为接驳线网中的重复站点.

\section{2 公交接驳线网优化模型}

FBNDP-SD 的具体优化变量为接驳线网 $\Omega$ 的公 交接驳线路条数 $K$, 以及每一公交接驳线路 $k(k=$ $1,2, \cdots, K)$ 的经由 $\omega_{k}$ 、接驳车站 $n_{P_{k}}$, 开行频率为 $f_{k}$ 、 各客流起论点 (origin-destination, OD) 的线路流量 $P_{i d}^{k}(i \in B, d \in T)$.

优化目标需同时考虑客流出行费用和运营企业 的线路开行成本. 乘客出行费用与其换乘路径上的 公交接驳线路和轨道交通换乘站有关. 乘客乘坐接 驳线路 $k$ 的候车费用和乘车费用分别为 $\frac{\lambda_{w}}{2 f_{k}} \sum_{d=I+1}^{I+J} \sum_{i=1}^{I} \sum_{h=1}^{I+J} P_{i d}^{k} X_{i h k}$ 和 $\frac{\lambda_{r}}{v_{B}}\left\{L_{k} \sum_{d=I+1}^{I+J} P_{n_{1}^{k} d}+\left(L_{k}-\right.\right.$ $\left.\left.L_{n_{1}^{k} n_{2}^{k}}\right) \sum_{d=I+1}^{I+J} P_{n_{2}^{k} d}+\cdots+L_{n_{P_{k}^{k}}^{k}-1_{P_{k}}} P_{n_{P_{k}}^{k}-1^{d}}\right\}$; 换乘费用为 $\sum_{j=I+1}^{I+J} \lambda_{j}\left(\sum_{d=I+1}^{I+J} \sum_{i=1}^{I} P_{i d}^{k} Y_{i j k}-P_{i j}^{k}\right)$; 乘客通过 $k$ 换乘至轨道 交通列车的乘车费用为 $\frac{\lambda_{T}}{v_{T}}\left(\sum_{d=I+1}^{I+J} \sum_{j=I+1}^{I+J} \sum_{i=1}^{I} P_{i d}^{k} Y_{i j} L_{j d}\right)$; $\lambda_{w} 、 \lambda_{r}$ 及 $\lambda_{T}$ 为对应货币费用转换系数; $\lambda_{j}$ 为接驳轨 道车站的每人次换乘费用.

公交运营企业线路 $k$ 的开行成本按双向计算为 $2 \lambda_{b} f_{k} \sum_{h=1}^{I+J} \sum_{i=1}^{I} L_{i h} X_{i h k}$, 其中, $\lambda_{b}$ 为单位车辆每公里的 运营成本. 
包含乘客广义出行费用和运营者运营成本的系 统费用最小化优化目标函数为

$$
\min Z(\Omega)=\min \sum_{k=1}^{K} Z^{k}
$$

其中, $Z^{k}$ 为公交接驳线路 $k$ 相关的系统费用，即

$$
\begin{aligned}
& Z^{k}=\frac{\lambda_{T}}{v_{T}}\left(\sum_{d=I+1}^{I+J} \sum_{j=I+1}^{I+J} \sum_{i=1}^{I} P_{i d}^{k} Y_{i j k} L_{j d}\right)+ \\
& \frac{\lambda_{w}}{2 f_{k}} \sum_{d=I+1}^{I+J} \sum_{i=1}^{I} \sum_{h=1}^{I+J} P_{i d}^{k} X_{i h k}+ \\
& \frac{\lambda_{r}}{v_{B}}\left\{L_{k} \sum_{d=I+1}^{I+J} P_{n_{1}^{k} d}+\left(L_{k}-L_{n_{1}^{k} n_{2}^{k}}\right) \sum_{d=I+1}^{I+J} P_{n_{2}^{k} d}+\cdots+\right. \\
& \left.L_{n_{P_{k}}^{k}-1^{n_{p_{k}}}} P_{n_{P_{P_{k}}}^{k}-^{d}}\right\}+\sum_{j=I+1}^{I+J} \lambda_{j}\left(\sum_{d=I+1}^{I+J} \sum_{i=1}^{I} P_{i d}^{k} Y_{i j k}-P_{i j}^{k}\right)+ \\
& 2 \lambda_{b} f_{k} \sum_{h=1}^{I+J} \sum_{i=1}^{I} L_{i h} X_{i h k}
\end{aligned}
$$

对于客流 $P_{i d}$, 若其起点 $i$ 位于线路 $k$ 上, 即 $\sum_{h=I+1}^{I+J} Y_{p h k}=1$ 时, 设 $n(i, k)$ 为 $i$ 在 $k$ 上的站点序号, 则客流 $P_{i d}$ 选择线路 $k$ 出行的费用为

$$
\begin{array}{r}
Z_{i d}^{k}=\lambda_{T} Y_{i n_{p k} k} L_{n_{p k} d} / v_{\mathrm{T}}+\lambda_{w} \sum_{h=1}^{I+J} X_{i h k} / 2 f_{k}+ \\
\lambda_{r} / v_{B} \sum_{p=n(i, k)}^{n_{p_{k}-1}} L_{n_{p h} n_{p k+1}}+\lambda_{j}\left(1-Y_{i d k}\right)
\end{array}
$$

客流 $P_{i d}$ 选择线路 $k$ 的客流量与换乘网络路径 费用有关. 以 Logit 分配作为客流选择函数，则该 $\mathrm{OD}$ 客流在线路 $k$ 上的分配客流量为

$$
P_{i d}^{k}=\frac{f_{k} \mathrm{e}^{-\lambda Z_{l d}^{k}} \sum_{j=1}^{I+J} Y_{i j k}}{\sum_{k^{\prime}=1}^{K}\left(f_{k^{\prime}} \mathrm{e}^{-\lambda Z_{l i}^{k^{\prime}}} \sum_{j=1}^{I+J} Y_{i j k^{\prime}}\right)} P_{i d}
$$

基于需求可拆分的公交接驳线网优化模型由目 标函数 (12) 和约束条件 (1) 至 (11)、客流选择函数 (15) 以及关联函数 (13) 和 (14) 组成. 与 FBNDP 优 化模型相比, 除网络结构约束变化外, 还增加了线 路间客流选择函数 $(15)$.

在模型中, 对于接驳线路 $k$ 的最优开行频率 $\bar{f}_{k}^{*}$ 可根据式 (13) 求解 $Z^{k}$ 关于 $f_{i j}$ 的一阶最优条件, 并 考虑约束条件 (10) 求得

$$
\bar{f}_{k}^{*}=\max \left\{\frac{1}{2} \sqrt{\lambda_{w} P_{k} / \lambda_{b} L_{k}}, P_{k} /(C \rho)\right\}
$$

路长度. 相应的线路费用为

$$
\begin{aligned}
C_{k}= & \frac{\lambda_{T}}{v_{T}}\left(\sum_{i=n_{1}^{k} d=I+1}^{n_{P_{k}-1}^{k}} \sum^{I+J} P_{i d}^{k} L_{n p_{k} d}\right)+\lambda_{w} P_{k} / 2 f_{k}+ \\
& \lambda_{n p_{k}}\left(P_{k}-\sum_{i=n_{1}^{k}}^{n_{P_{k}-1}^{k}} P_{i n p_{k}}\right)+2 \lambda_{b} f_{k} L_{k}+\lambda_{r}\left\{L_{k} \sum_{d=I+1}^{I+J} P_{n_{1}^{k} d}+\right. \\
& \left.\left(L_{k}-L_{n_{1}^{k_{n} n_{2}^{k}}}\right) \sum_{d=I+1}^{I+J} P_{n_{2}^{k} d}+\cdots+L_{n_{P_{k-1}^{k}}^{k} n_{P_{k}}} P_{n_{P_{k-1}^{k}}^{k} d}\right\} / v_{B}
\end{aligned}
$$

\section{3 模型求解}

FBNDP 具有多个变量及约束条件, 求解方法多 采用启发式算法 ${ }^{[1]}$, FBNDP-SD 较 FBNDP 更难求 解, 本研究采用遗传算法 ${ }^{[15]}$ 进行求解. 由于 FBNDP-SD 问题构解较为困难, 在初始种群个体生 成和交叉操作生成子代个体过程中, 均是先生成 FBNDP 线网, 再以此为基础生成 FBNDP-SD 线网.

对初始种群个体生成操作, 首先通过选取公交 站点不断扩充公交线网, 形成不包含重复点的初始 个体, 再插人重复点形成 FBNDP-SD 个体, 构成初 始接驳线网种群, 并根据客流选择规律在接驳线路 间分配客流量和公交线路开行频率. 对于交叉操 作, 针对父代双亲个体, 剔除重复站点形成 FBNDP 个体, 采用与文献 $[5]$ 类似方法双亲单子生成 2 个 FBNDP 子代个体, 并将剔除的重复站点按照一定插 人策略随机插人 FBNDP 子代个体, 完成 FBNDP-SD 子代个体的构造. 具体算法流程见图 1.

\section{1 编码方案和适应度}

公交接驳线网问题一般采用自然数编码, 即采 用公交车站与轨道交通车站编号表示, 使站点与基 因片段对应. 如当 $B=\{1,2,3,4,5,6,7\}, T=\{8$, $9,10\}$ 时, 某一接驳线网表示为 12384596710 , 该接驳线网由 3 条接驳线路组成, 对应上述 3 个字 串 $1238 、 459$ 及 6710 .

以最小化目标函数的倒数为基础, 考虑约束条 件 (11) 并引入罚因子 $\lambda$, 构造个体 $\Omega$ 的适应度函 数为

$$
\begin{aligned}
F(\Omega)= & \frac{1}{Z(\Omega)}+ \\
& \lambda\left[\frac{1}{2} N v_{B}(T-t)-\sum_{k=1}^{K} f_{k} \sum_{i=1}^{I} \sum_{h=1}^{I+J} L_{i h} X_{i h k}\right]
\end{aligned}
$$




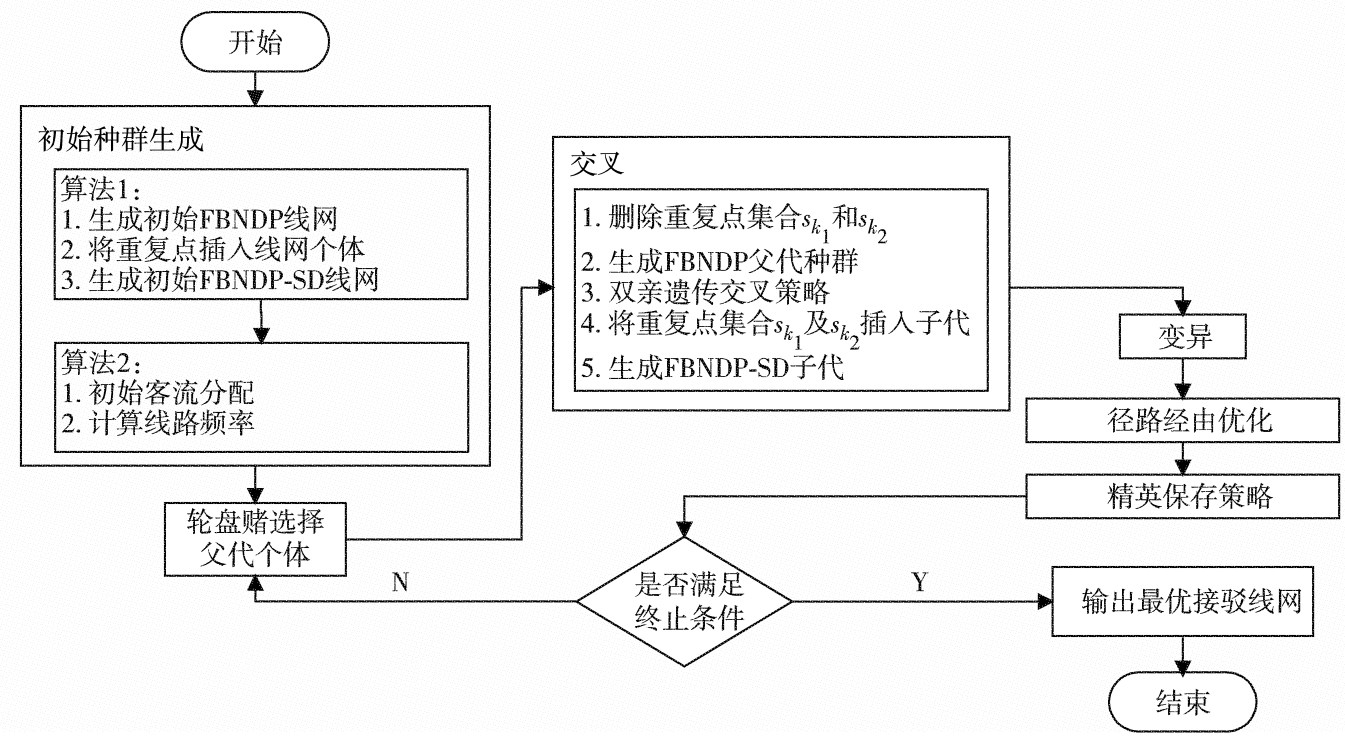

图 1 算法流程图

Fig. 1 Framework of the proposed algorithm

\section{2 初始种群生成}

随机选择轨道车站, 按照一定概率选择未在线 网中的公交车站, 将其加人该接驳车站所属的某条 线路, 或由该轨道车站和公交站点组成新的直连接 驳线路. 对于轨道车站 $j$ 和公交车站 $i$ 构成的直连接 驳线路, 可依据式 (13) 和式 (16), 获得线路的最 优系统成本 $\mathrm{DC}_{i j}$ 为

$$
\begin{aligned}
\mathrm{DC}_{i j}= & \frac{\lambda_{T}}{v_{T}}\left(\sum_{d=I+1}^{I+J} P_{i d}^{k} L_{j d}\right)+\lambda_{w} \sum_{d=I+1}^{I+J} P_{i d}^{k} / \bar{f}_{i j}^{*}+ \\
& \lambda_{r} L_{i j} \sum_{d=I+1}^{I+J} P_{i d}^{k} / v_{B}+\lambda_{j}\left(\sum_{d=I+1}^{I+J} P_{i d}^{k}-P_{i j}^{k}\right)+ \\
& 2 \lambda_{b} \bar{f}_{k}^{*} L_{i j}
\end{aligned}
$$

令 $\mathrm{DC}_{i j}^{*}=\max _{i \in B} \mathrm{DC}_{i j}+\operatorname{minDC}_{i \in B}$, 置公交车站 $i$ 和轨 道车站 $j$ 的直接连接线路评价函数为

$F_{i j}=\mathrm{DC}_{i j}^{*}-\mathrm{DC}_{i j}$

FBNDP 初始线网构建完毕后, 对每一公交站点 尝试作为重复点, 构造包含重复点的 FBNDP-SD 初 始线网.

算法 1 初始 FBNDP-SD 线网生成算法, 其步 骤为

1) 置集合 $B^{\prime}=B$ 为构建当前接驳线路的可选 择公交站集, $\Omega$ 为此时的接驳线网, 且线路编号 $k=1$.

$2)$ 若 $B^{\prime}=\phi$, 转 5). 否则, 从 $T$ 中选取 1 个轨 道车站 $j$, 选择方法为等概率随机选取.

3 ) 采用轮盘奢方法, 从 $B^{\prime}$ 选取 $I$ 次公交站点 $i$,
每个公交站点 $i$ 被选取的概率为 $F_{i j} / \sum_{i=1}^{I} F_{i j}$. 对轨道 车站 $j$, 每次按均匀分布生成区间 $[0,1]$ 内的随机数 $R$ 选择 1 个公交站点 $i$, 使 $\sum_{i^{\prime}=1}^{i-1} F_{i^{\prime} j} / \sum_{i^{\prime}=1}^{I} F_{i^{\prime} j} \leqslant R<$ $\sum_{i^{\prime}=1}^{i} F_{i^{\prime} j} / \sum_{i^{\prime}=1}^{I} F_{i^{\prime} j}$ 成立, 若 $R<F_{1 j}$, 则选择公交站点 1 .

4) 若不存在以 $j$ 为终点的接驳线路, 则以 $j$ 为 终点将 $i$ 与 $j$ 相连新建线路 $\omega_{k}, \Omega=\Omega \cup\left\{w_{k}\right\}, k=$ $k+1, B^{\prime}=B^{\prime} \backslash i$, 转 2$)$. 否则, 若共有 $T$ 条接驳线 路以 $j$ 为终点. 对于第 $t(t=1,2, \cdots, T)$ 条接驳线 路, 按 $\mathrm{DC}_{i j}$ 由小到大的顺序确定 $i$ 距离终点 $j$ 的位 置, 将 $i$ 逐个插人 $t$, 得到当前线网方案 $\Omega_{T}$; 以 $j$ 为 终点将 $i$ 与 $j$ 相连, 形成新线, 并将其加人 $\Omega$, 此时 接 驳线网为 $\Omega_{T+1}$. 假 设 $Z\left(\Omega^{\prime}\right)=$ $\min _{\Omega \in\left(\Omega_{t}(t=1,2, \cdots, T), \Omega_{T+1}\right)}\{Z(\Omega)\}$, 则 $\Omega=\Omega^{\prime}$. 若 $\Omega^{\prime}=$ $\Omega_{T+1}, k=k+1, B^{\prime}=B^{\prime} \backslash i$, 转 2). 否则, 若 $B^{\prime}=$ $B^{\prime} \backslash i$, 转 2). FBNDP 初始线网构造完毕则转 5).

5 ）对于已构造的 FBNDP 初始线网, 采取重复 点添加策略构造 FBNDP-SD 初始线网: 对于每个公 交站点 $i$, 分别找到距离其最近, 且不包含其本身的 线路加人 (以点到点之间的距离作为衡量), 使 $i$ 点同属于不同线路, 将线网转换为需求可拆分情形 并记录重复点 $i^{\prime}$ 集合.

对每一 FBNDP-SD 线网, 重复点在各条线路的 流量分配算法见算法 2.

算法 2 线路客流分配及频率确定算法, 其步 


\section{骤为}

1) 已知包含重复点的初始解, 其中的重复点 $i^{\prime}$ 为需要进行客流需求拆分的点.

2) 对于每个重复点 $i^{\prime}$ 采取以下处理: 将多条 经过 $i^{\prime}$ 点的线路 $k$ 中的重复点 $i^{\prime}$ 客流需求视为初始 强度，利用式(16)计算其在每条线路中的开行频率 $f_{k}^{1}, f_{k}^{2}, \cdots$, 并通过如式 (15) 的 Logit 分配进行客流 选择, 得到初始分配后重复点在各线路的客流 $P_{i d}^{k}$.

3) 已知第 1 次分配后重复点在各线路的客流 $P_{i d}^{k}$, 利用式 (16) 计算其在每条线路中的开行频率 $f_{k}^{1^{\prime}}, f_{k}^{2^{\prime}}, \cdots$, 并通过式 (15) 进行客流选择，得到分 配后重复点在每条线路的客流 $P_{i d}^{\prime k}$.

4) 循环步骤 3$)$, 直到该重复点 $i^{\prime}$ 在每条线路 上的客流比例趋于稳定，算法结束. 设 $x_{i^{\prime}}^{n}$ 及 $x_{i^{\prime}}^{n+1}$ 为 点 $i^{\prime}$ 在线路 $k$ 上相邻两次分配后客流量的比例, 并 满足条件 (19) 则结束 4), 其中, $I^{\prime}$ 为重复点集合; $\varepsilon$ 为预定精度参数.

$$
\sqrt{\sum_{i^{\prime} \in I^{\prime}}\left(x_{i^{\prime}}^{n+1}-x_{i^{\prime}}^{n}\right)^{2}} / \sum_{i^{\prime} \in I^{\prime}} x_{i^{\prime}}^{n} \leqslant \varepsilon
$$

采用算法 1 ，每次构造 1 个个体，直至达到初 始种群规模 $n$ 为止.

\section{3 遗传算子设计}

\section{3 .1 选择复制}

采用 2 种机制增强算法搜索能力: (1) 竞争机 制. 复制 $n$ 个个体的种群以产生 $2 n$ 个个体的种群, 两两比较随机分成 $n$ 对后的个体适应度，保留较优 的种群规模个体; (2) 人侵机制. 采用初始种群生 成算法产生 $n$ 个个体并找到其中适应度最高的 $\rho n$ 个 个体，用于替代上述个体中适应度最低的 $\rho n$ 个个 体, 其中, $\rho \in(0,1)$ 为人侵比例, 可以提前设定 为常数或采用动态控制策略.

\section{3.2 交叉操作}

对选中的父代个体，采用双亲双子的交叉操 作，其步骤为

1) 剔除双亲的重复点，形成 FBNDP 个体. 具 体对于每个重复点，比较其在各线路上的客流量， 保留客流量最大的重复点并剔除该点在其他线路中 的重复点.

2) 对已剔除重复点的 FBNDP 父代双亲个体, 分别以某一父代个体为基础, 采用文献 $[2]$ 的双亲 单子交叉操作, 对于 FBNDP 父代个体 $\Omega_{l_{1}}$ 及 $\Omega_{l_{2}}$, 以 $\Omega_{l_{1}}$ 为基础, 以 $\Omega_{l_{1}}$ 的首个基因位对应的公交站为起 点, 按顺序比较其在两个父代中下一个站点与该线 路接驳轨道车站间的线路费用，保留较好点作为新
生子代的基因, 并以之为起点继续比较. 在 $\Omega_{l_{1}}$ 和 $\Omega_{l_{2}}$ 中去除该基因，构造当前线路的结束条件为选 取基因对应轨道车站. 对所有公交站点重复此过 程, 形成子代.

3) 将两个父代个体的重复点按概率插人两个 FBNDP 子代个体，形成 FBNDP-SD 子代个体. 将父 代个体中的重复点作为集合，将集合中的每个重复 点随机插人其中一个子代个体（为保证插人重复点 的科学性和效率性, 插人位置为该点所在线路外的 线路上，最靠近该点的 5 个公交点的位置). 对重 复点的各个备选插人位置, 通过调用算法 2 计算各 线路的客流量及频率，并优化备选重复点所在线路 的径路经由，接受使线网费用下降程度最大的插人 方案.

\subsection{3 变异操作}

为增强全局寻找最优解能力, 以变异概率 $P_{m}$ 随机选取基因点, 并依据概率 $p$ 接受较差变异解. 若选中公交站点，将其随机插人到其他公交站点对 应的基因位; 若选中轨道接驳车站, 则随机选择另 一轨道接驳车站替代. 对变异解需考虑其变异质 量, 即接受概率为 ${ }^{[16]}$

$$
p=\left\{\begin{array}{cc}
1, & \Delta Z<0 \\
\mathrm{e}^{-\Delta Z / Z_{0}}, & \Delta Z \geqslant 0
\end{array}\right.
$$

其中, $Z_{0}$ 为此时最高适应度个体的适应度值; $\Delta Z$ 为 变异前后的目标差值. 对每个个体的变异操作为 $\left\lfloor\frac{I+J}{2}\right\rfloor$ 次 $^{[17]}$.

\section{4 辅助优化策略}

1）径路经由优化策略. 对交叉操作中的子代 个体，其每一条接驳线路均需优化其径路经由. 单 条接驳线路的径路经优化后均可视为一个具有固定 终点的车辆路径问题. 采取在线路内部随机挑选 2 个公交站点 $i$ 和 $j$ 进行单点交换的方法逐步优化线 路径路.

2) 精英保存策略. 每次产生新一轮满足种群 规模的子代后, 将本轮最优解与历史最好解比较并 保留二者中较优解.

\section{5 终止条件}

算法终止于达到最大进化代数 $T_{\text {max }}$ 或目标函数 值在 $T^{\prime}$ 代内收敛.

\section{4 算例分析}

采用文献 $[5]$ 构造的通用算例网络，包含 80 个 
公交站点和 4 个轨道车站. 遗传算法中设置种群规 模为 100 , 交叉概率为 0.8 , 变异概率为 0.08 , 最 优个体保持代数为 120 , 最大进化代数为 1500 . 采 用 $\mathrm{C}$ \#语言编程, 并分析不同客流需求强度下的优 化结果. 模型参数见表 1 .

\section{表 1 模型参数}

Table 1 Model parameters

\begin{tabular}{|c|c|c|c|}
\hline 参数 & 取值 & 参数 & 取值 \\
\hline$C /$ 人 & 50 & $\lambda_{T} /\left(\right.$ 元 $\cdot$ 人 $\left.^{-1} \cdot \mathrm{h}^{-1}\right)$ & 16 \\
\hline$\rho$ & 1.2 & $\lambda_{j} /\left(\right.$ 元 $\cdot$ 人 $\left.^{-1}\right)$ & 0.2 \\
\hline$t / \mathrm{h}$ & 0.16 & 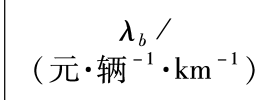 & 18 \\
\hline$N /$ 辆 & 110 & $V_{B} /\left(\mathrm{km} \cdot \mathrm{h}^{-1}\right)$ & 20 \\
\hline$\lambda_{w} /\left(\right.$ 元 $\cdot$ 人 $\left.^{-1} \cdot \mathrm{h}^{-1}\right)$ & 24 & $V_{T} /\left(\mathrm{km} \cdot \mathrm{h}^{-1}\right)$ & 36 \\
\hline$\lambda_{r} /\left(\right.$ 元 $\cdot$ 人 $\left.^{-1} \cdot \mathrm{h}^{-1}\right)$ & 12 & $\lambda$ & 0.0001 \\
\hline
\end{tabular}

\section{1 固定客流强度下的优化结果}

考虑每个公交站点与各轨道车站均有客流需求 且客流分布均等. 单位时段内公交站的客流需求强 度为 200 人. 最优接驳线网如图 1. 其中, 接驳线 路数 22 条, 平均长度 $1.70 \mathrm{~km}$. 线路每小时开行频 率为 13.54. 接驳线网方案存在 1 个重复点, 为 40 号站点. 系统总费用为 53273 元. 旅客乘坐公交时 间与旅客乘坐列车时间比值为 $1.20: 0.81$, 旅客绕 行比为 1.25 .

FBNDP-SD 最优线网与文献 [5] 中 FBNDP 结果
的对比见表 2 , 系统总费用的降低了 $0.03 \%$ 。可 见，在相同线网规模及轨道车站客流分布等条件 下，允许需求可拆分情形时，接驳线网方案更为优 化合理, 线路长度略有减少, 但开行频率维持不 变. 由于问题的复杂性大大增加，使得运算时间显 著增加.

表 2 需求拆分与否条件下的最优接驳线网对比

Table 2 Comparison between best feeder-bus network without split delivery and with split delivery

\begin{tabular}{cccccc} 
是否需 最优解接 系统总费用/ 线路平均 平均开 平均运算 \\
求拆分 驳线路数 \\
$\begin{array}{c}\left(\text { 元 } \cdot \mathrm{h}^{-1}\right) \\
\text { 是 }\end{array}$ & 22 & 53273 & 1.70 & 13.54 & 1122 \\
否 & 22 & 53292 & 1.72 & 13.54 & 52 \\
\hline
\end{tabular}

\section{2 站点客流需求差异的优化结果}

上述算例中每个公交站点客流需求强度在接驳 线网中的地位一致, 且公交站点客流需求在轨道交 通站点之间均匀分布，接驳线路交叉关系并不明 显. 为对比不同客流需求下的线网结果，对公交站 点采取在 $50 \sim 400$ 人按 50 的倍数随机设置单位时 段客流需求，并且每个公交站点与轨道交通站点间 的客流呈线路两头大中间小的不均匀分布，与 4 个 轨道交通车站间客流比例分别为 $40 \% 、 10 \% 、 10 \%$ 及 $40 \%$ ，考察 FBNDP-SD 线网的变化规律.

求得的最优接驳线网如图 2 与图 3 (站点编号 右下角标表示客流需求). 其中，接驳线路 24 条，

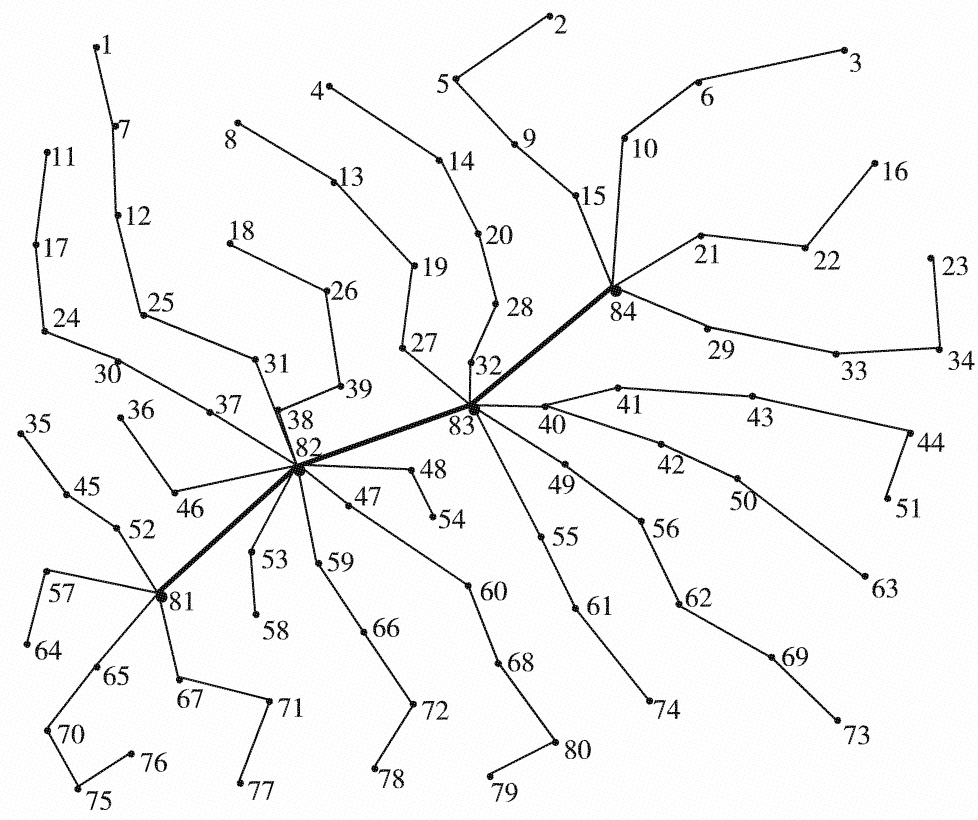

图 2 固定客流强度下的最优接驳线网

Fig. 2 Best result under constant flow volume 


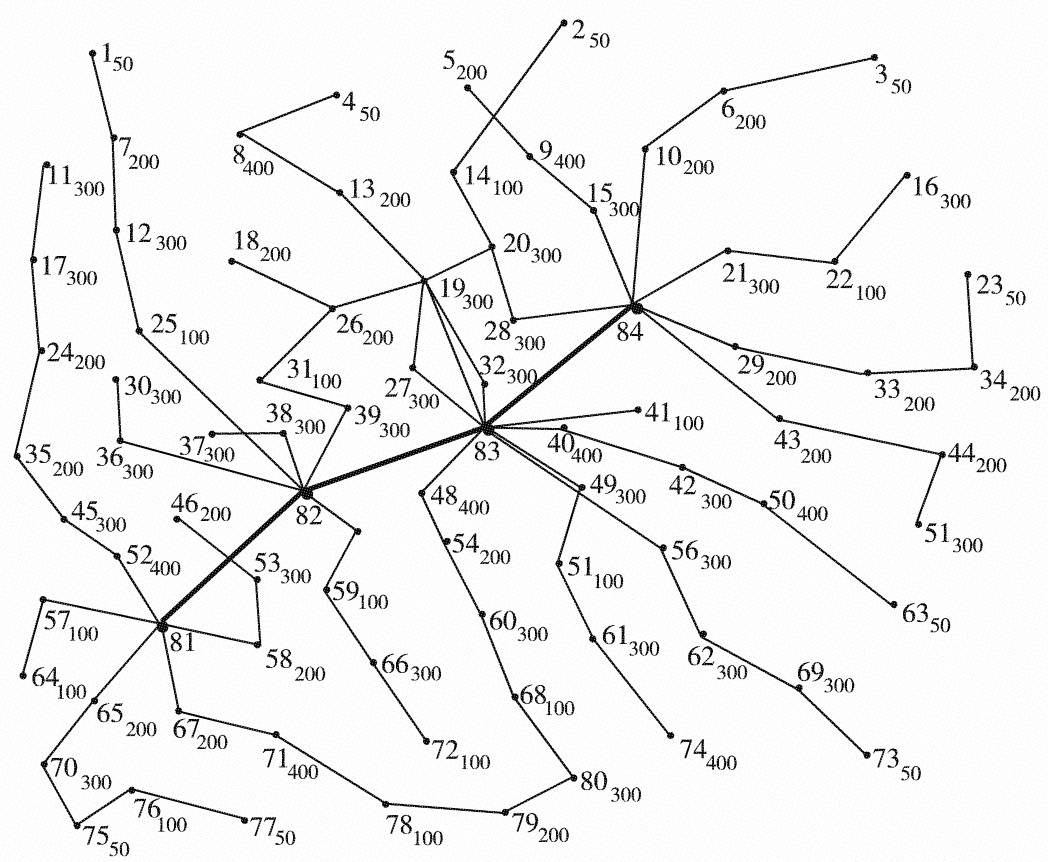

图 3 站点客流需求差异的最优接驳线网

Fig. 3 Best result under varying flow volume

平均长度为 $1.74 \mathrm{~km}$. 线路每小时开行频率为 13. 96 . 系统总费用为 60980 元. 接驳线网方案存 在 8 个重复点, 分别是 $26 、 19 、 20$ 和 79 号站点, 其中, 19 号公交站点重复 5 次, 这些站点的客流需 求均为 200 人或 300 人.

与固定客流强度下的优化结果相比, 重复点的 数量随整体网络客流强度的增大由 1 个增加到 8 个, 重复站点设置更倾向于客流较多的站点.

在相同线网条件下，需求可拆分情况下 FBNDP-SD 最优线网与不考虑需求可拆分条件的 FBNDP 最优线网各项指标对比见表 3 . 其中，表 3 第 2 行表示不考虑需求可拆分条件的公交接驳最优 线网结果. 通过表 3 可知, 系统总费用降低比率达 到 $0.61 \%$ ，与固定客流强度下的优化结果 $(0.03 \%)$ 对比, 费用降低效果更为明显，且线路平

表 3 需求拆分与否条件下的最优接驳线网各项指标对比

Table 3 Comparison between best feeder-bus network without split delivery and with split delivery

\begin{tabular}{ccccc}
\hline $\begin{array}{c}\text { 是否需 } \\
\text { 求拆分 }\end{array}$ & 线路数 & $\begin{array}{c}\text { 系统总费 } \\
\text { 用 } /\left(\text { 元 } \cdot \mathrm{h}^{-1}\right)\end{array}$ & $\begin{array}{c}\text { 线路平均 } \\
\text { 长度 } / \mathrm{km}\end{array}$ & $\begin{array}{c}\text { 平均开 } \\
\text { 行频率 }\end{array}$ \\
\hline 是 & 24 & 60980 & 1.74 & 13.96 \\
否 & 24 & 61355 & 1.84 & 13.78 \\
\hline
\end{tabular}

均长度减少, 开行频率略有上升. 考虑需求可拆分 情形可降低线路的平均长度，为乘客提供更多的线 路选择，说明在客流需求量大的情况下，允许需求 拆分、多条线路共同为某些需求量大的站点提供服 务, 对降低网络费用、优化线网结构更具实际意义.

\section{结语}

本研究针对经典 FBNDP, 进一步放宽假设条 件，允许需求在线路间拆分，使公交站点可以为多 条公交接驳线路服务，建立基于换乘网络且满足需 求可拆分的公交接驳线网优化模型. 根据模型特点 设计了基于遗传算法的求解方法. 模型和算法综合 考虑需求可拆分条件下的网络结构和客流选择行为 等约束条件; 在优化算法中，以 FBNDP 的解为基 础, 融人重复点的生成和插入策略, 实现 FBNDP 解和 FBNDP-SD 解的相互转换, 有效解决了 FBNDP-SD 构解困难的问题. 优化结果表明，在考 虑公交需求可拆分的情形下，FBNDP-SD 线网能够 为公交站点提供多条接驳线路进行选择，从而有效 分流乘客，降低线路成本，优化线网结构. 对于线 网客流强度较高的情况或者具有较大客流量的站 点，考虑公交需求可拆分更具实际意义. 
基金项目: 国家自然科学基金资助项目 (71471179, U1834209)

作者简介: 邓连波 $(1977-)$, 中南大学教授、博士生导师. 研究 方向：交通运输运营管理、公共交通组织优化. E-mail: lbdeng@ csu.edu.cn

引文：邓连波，何 渊，曾俊豪，等. 需求可拆分下城轨关联 的公交接驳线网优化 $[\mathrm{J}]$. 深圳大学学报理工版, 2020 , $37(2): 121-129$.

\section{参考文献 / References:}

[ 1 ] KUAH G K, PERL J. The feeder-bus network-design problem $[\mathrm{J}]$. Journal of the Operational Research Society, $1989,40(8)$ : 751-767.

[ 2 ] BYRNE B F, VUCHIC V R. Public transportation line positions and headways for minimum cost [ DB/OL]. (1973-12-22) [2018-10-11 ]. https ://trid. trb. org/view. aspx id $=132894$.

[ 3 ] LENSTRA J K, KAN A H. Complexity of vehicle routing and scheduling problems [J]. Networks, 1981, 11 (2) : 221-227.

[ 4 ] KUAN S N, ONG H L, NG K M. Solving the feeder bus network design problem by genetic algorithms and ant colony optimization [ J ]. Advances in Engineering Software, 2006, 37(6) : 351-359.

[ 5 ] 邓连波, 高 伟, 赖天珍, 等. 基于换乘网络的城市 轨道关联公交接驳线网优化 $[\mathrm{J}]$. 铁道科学与工程学 报, 2012,9(6): 77-83.

DENG Lianbo, GAO Wei, LAI Tianzhen, et al. Optimal design of feeder-bus network related to urban rail transit based on transfer network $[\mathrm{J}]$. Journal of Railway Science and Engineering, 2012, 9(6) : 77-83. (in Chinese)

[ 6 ] LI Xin, WEI Ming, HU Jia, et al. An agent-based model for dispatching real-time demand-responsive feeder bus $[\mathrm{J}]$. Mathematical Problems in Engineering, 2018, 2018(1): 1-11.

[ 7 ] SUN Bo, WEI Ming, YANG Chunfeng, et al. Personalised and coordinated demand-responsive feeder transit service design: a genetic algorithms approach $[\mathrm{J}]$. Future Internet, 2018, 10(7): 61-74.

[ 8 ] ANASTASIOS C, CHRISTINA L, KONSTANTINOS K. DRT route design for the first/last mile problem: model and application to Athens, Greece $[\mathrm{J}]$. Public Transport, 2018, 10(3): 499-527.

[ 9 ] TAPLIN J H, SUN Yuchao. Optimizing bus stop locations for walking access: stops-first design of a feeder route to enhance a residential plan $[\mathrm{J}]$. Environment and Planning B: Urban Analytics and City Science, 2019: 1-23.

[10] 刘新宇, 符 卓, 邱 萌. 需求可拆分车辆路径问题
研究 $[\mathrm{J}]$. 技术经济，2017，36 (1)：96-108.

LIU Xinyu, FU Zhuo, QIU Meng. Research on vehicle routing problem with split delivery: literature review $[\mathrm{J}]$. Technology Economics, 2017, 36(1) : 96-108. (in Chinese)

[11] JIN Mingzhou, LIU Kai, BRUAK E. A column generation approach for the split delivery vehicle routing problem [J]. Operations Research Letters, 2008, 36(2) : 265270.

[12] 李三涁, 柴玉梅, 王黎明. 需求可拆分的开放式车辆 路径问题研究 $[\mathrm{J}]$. 计算机工程, 2011, 37(6)：168171.

LI Sanbin, CHAI Yumei, WANG Liming. Research on split delivery open vehicle routing problem $[\mathrm{J}]$. Computer Engineering, 2011, 37(6) : 168-171. (in Chinese)

[13] KHMELEY A, KOCHETOY Y. A hybrid VND method for the split delivery vehicle routing problem $[\mathrm{J}]$. Electronic Notes in Discrete Mathematics, 2015, 47: 5-12.

[14] 符 卓, 刘 文, 邱 萌. 带软时间窗的需求依订单 拆分车辆路径问题及其禁忌搜索算法 $[\mathrm{J}]$. 中国管理 科学, 2017, 25(5): 78-85.

FU Zhuo, LIU Wen, QIU Meng. A tabu search algorithm for the vehicle routing problem with soft time windows and split deliveries by order $[\mathrm{J}]$. Chinese Journal of Management Science, 2017, 25 (5) : 78-85. (in Chinese)

[15] 陈国良, 王煦法, 庄镇泉, 等. 遗传算法及其应用 $[M]$. 北京: 人民邮电出版社, 1996.

CHEN Guoliang, WANG Xufa, ZHUANG Zhenquan, et al. Genetic algorithm and its application $[\mathrm{M}]$. Beijing: Posts \& Telecom Press, 1996. (in Chinese)

[16] 邓连波, 史 峰, 莫辉辉. 物流配送车辆路径问题多 代竞争遗传算法 $[\mathrm{J}]$. 铁道科学与工程学报, 2005, 2 (5) : 75-79.

DENG Lianbo, SHI Feng, MO Huihui. Multi-generation compete genetic algorithms for logistics distribution vehicle routing problem $[\mathrm{J}]$. Journal of Railway Science and Engineering, 2005, 2(5): 75-79. (in Chinese)

[17] 符 卓, 聂 靖. 求解带装载能力限制的开放式车辆 的路径问题的遗传算法 $[\mathrm{J}]$. 系统工程, 2008, 26 (2) : 78-83.

FU Zhuo, NIE Jing. A genetic algorithm for the capacitated open vehicle routing problem $[\mathrm{J}]$. Systems Engineering, 2008, 26(2) : 78-83. (in Chinese)

【中文责编：方 圆; 英文责编：淡 紫】 